\title{
El valor arqueológico del Museo San Pedro de Atacama
}

Es bastante difícil al autor de esta presentación del primer número de una nueva revista Estudios Atacameños, ubicar su punto de vista por varias razones.

La primera es que él mismo es el creador de los estudios atacameños, como también del museo donde están conservadas todas las pruebas de estas investigaciones.

Además, una nueva revista podría hacer pensar que no tiene antecedentes. Es lo contrario, mucho ya está publicado acerca de la Cultura Atacameña, especialmente en los Anales de la Universidad del Norte en sus números desde 1961, en la Revista Universitaria de la Universidad Católica de Santiago desde 1958, y en los Anales de la Universidad Católica de Valparaíso en 1957-8.

La tercera razón consiste en la necesidad de presentar a los lectores de la nueva revista un resumen de lo ya realizado para unirlo a las perspectivas y proyectos para el futuro en conexión con lo que se efectúa en el momento actual.

Creemos que lo más fácil y más objetivo consiste en una nomenclatura o una lista de sitios, de hallazgos, de material, cosa sin vida por desgracia, pero que hará entender de inmediato la suma de trabajos y estudios en realización.

Este resumen se presenta en cuatro categorías:

1. Los sitios del Paleolítico o de la piedra tallada, que unos llaman precerámico, en sus tres épocas o evoluciones distintas con el material recogido y ya estudiado o en estudio.

2. Los "hábitat", pueblos, estancias, con vestigios de construcciones, muros, pircas en ruinas. También éstos serán divididos en varias épocas, empezando en el Paleolítico para terminar en las construcciones españolas. A los cuales hay que agregar los sitios con petroglifos.
3. Los cementerios con sus 5.100 tumbas salvadas y catalogadas, el estudio del Indice neo de 80 de las 150 momias, o mejor dicho "cuerpos deshidratados".

4. Una nomenclatura breve y general, sin entrar en los detalles propios de estudios detenidos, de cada categoría de objetos, del material de la época neolítica, o agroalfarera, conservado en el Museo de San Pedro de Atacama.

Antes de dar a conocer estas cuatro listas nos permitirán recordar que hemos tenido en el $\mathrm{Mu}$ seo de San Pedro de Atacama ya dos congresos internacionales de arqueología, en 1963 y 1969, y avisar también que hemos empezado la publicación de una serie de cinco (o más) tomos. El primero ya apareció: Industrias líticas de San Pedro de Atacama. El segundo Secuencias líticas de San Pedro de Atacama y zonas limítrofes está en la imprenta. El tercero y cuarto tratarán del Neolítico y el quinto, de la Conquista española hasta hoy día, con nuestras conclusiones acerca de todos nuestros estudios atacameños.

1. Lista de los sitios del Paleolítico con el número de artefactos, conservados en el Museo de San Pedro de Atacama, hasta la fecha 15 de agosto de 1973

\section{Región Salitrera}

1. Oficina Altamira 4.876

2. Ovalo 22.204

3. Chicote 1.478

4. Cachina 299

5. Jurjillo 102

6. Baquedano $1 \quad 13.344$

7. Baquedano 2 (NE) $\quad 1.100$

8. Salar del Carmen 69

9. Estación Prat 192

10. Pampa Unión 8.912

11. Chacabuco 2.000

$\begin{array}{lr}\text { 12. Cerro Empinado } & \frac{837}{59.473} \\ \text { Total Región Salitrera }\end{array}$ 
2. Salar de Talabre-Río Loa.

1. Talabre

2. Salar W. Rudolph

3. Chuqui

4. Isla Grande

5. Zona de Mesetas

6. Salar Brinkerhoff

7. Cerro Salas

8. Chiu Chiu

9. Cerro Le Paige

10. Santa Bárbara (4 sitios)

11. Cueva de la Damiana

12. Conchi Viejo

13. Lequena (2 sitios) Total

\section{Tchasquir.}

1. Tchasquir

4. Ghatchi.

1. Loma 1

2. Loma 2

3. Loma 3

4. Loma 4

5. Loma 5

6. Loma 5 bis

7. Loma 5 ter

8. Loma 6

9. Loma 7

10. Loma 8

11. Loma 9

12. Loma 10

13. Loma 11

14. Loma 12

15. Loma 13

16. Loma 14

17. Loma 15

18. Loma 16

19. Loma 16 bis

20. Loma 17

21. Loma 18

22. Loma 19

23. Loma 20

24. Loma 21

25. Loma 22

26. Loma 22 bis

27. Loma 23

28. Loma 24

29. Loma 25

30. Loma 25 bis

17.098

8.549

1.863

3.600

9.085

3.005

155

164

152

61

$\frac{24}{46.823}$

583

20
31. Loma 26

698

32. Loma 27

3.289

33. Ghatchi 1

34. Ghatchi 2

4.169

35. Loma 28

55

36. Loma 28 bis

86

37. Loma 29

380

38. Loma 30

29

39. Camino del Inka a Chaxas

1. planicie baja

254

2. subida oriental

459

40. Ouebrada hacia planicie baja del

Camino del Inka

3

41. Loma Oriental norte cumbre aislada NE 9

42. Parte superior donde se juntan las dos lomas

182

43. Loma Oriental:

1. extremidad septentrional

2. planicie baja al $\mathrm{N}$ de la extremidad $\mathrm{N}$

3. larga subida al sur de la quebrada central

4. extremidad meridional cumbre

351

5. extremidad oriente pendiente 36

6. extremidad subida Vilama 639

44. Loma Occidental:

1. Centro

2. Meridional

103

3. Valle río Vilama

331

4. Calar (ruinas)

2.272

5. Calar alrededores

668

6. Calar orilla occidental

434

7. Parte NO

434

45. Mesa Central

402

46. Cumbre más septentrional (en piedras pomas) dirección Guatin

47. Artefactos sueltos

5. Chaxas.

48. Chaxas

49. Portezuelo Chaxas

50. Coral Bario

51. Ara Blanca

52. Cueva Pintada

53. Ojo por Arriba

54. Cerca del Arenal

55. Cuevita de Abajo

56. Agua Chitita

57. Callejón del Cerro Sali

58. Alto de la Vega 424

59. Camino del Laura $1 \quad 416$

60. Camino del Laura 2 
61. Mesada

62. Crucijada

63. Inca

6. Loma Negra.

64. Loma Negra

65. Fundiciones

7. Puripica.

66. Puripica

67. Vilama

68. Vilama

1. Parte baja

2. Planta azufre

3. Ruinas

68. Vilama
4. Subida Huella

5. Subida Huella

69. Linzor

70. Cerro Sayes

71. Cartuche

72. Incapite

73. Ghatchi 1 (ruinas)

74. Ghatchi 2 (ruinas)

75. Calar antes del pueblo

8. Guatin.

76. Chorro

77. Kesa

78. Entrada opuesta a Puripica

\section{Zona Ghatchi-Loma Negra-Guatin}

79. Lugar de subida del Lincabur entre Chaxas y Cajón

80. Carampa

81. Incaliri

82. Lincabur

83. Lincabur. Parte Occidental Cuevita

84. Faldeo Puripico

85. Yanada

86. Ronque

87. Puripite

88. Peña Colorada, mal paso alto de Puripite.

89. Escalera

90. De Catarpe a Caire

91. Caire 1

92. Caire 2

93. Caire 3
94. Catarpe (Orilla Oriental Meseta)

95. Quitor 1 (por arriba)

96. Quitor 5 (oriente) 28

97. Quitor 5 (sur) 25

98. Quitor Loma Planta Purico 146

99. Quitor $7 \quad 284$

100. Conde Duque 6

101. Tulor 36

102. Yona 1

103. Yona 2

104. Tebenquiche 153

105. Campo Solor 7

106. Campo Coyo 945

107. Tchaputchayna 935

Zona Norte (Zona central) y Salares del Norte.

108. Río Grande 22

109. Lari 73

110. Entre Lari y Caspana 10

111. Entre Catarpe y Pelún 90

112. Pelún $\quad 10.909$

113. Pelún Mutucuro 971

114. Faldeo Meridional 121

115. Pelún origen de la fuente 21

116. Pelún dirección de Caire 45

117. Peña Blanca 33

118. Tolún 148

119. Peñaliri 343

120. Envidia (cerca de Peñaliri) 96

121. Puritama 25

122. Apacheta (Machuca) 860

123. Apacheta norte $\quad 169$

124. Machuca $\quad 3.545$

125. Machuca Cerrito Blanco 112

126. Callejón 31

127. Machuca pie del cerro N E 106

128. Incahuasi (entre Machuca y el Tatio) 86

129. Cotorpuri 8

130. Tatio Geysers 39

131. Tatio fuente del río Salado 11

132. Incahuasi. Parte occidental río Putana 99

133. Putana 66

134 Fuente del Diablo 3

135. Ayquina (cerca Turi) $\quad 1.190$

136. Ayquina 1 (sifón) 148

137. Ayquina 2 (sifón) 46

138. Paguayte 126

139. Pila 92

140. Toconce: última colina $\mathrm{O} \quad 6$

141. Ojos de San Pedro 117

142. Ojos de San Pedro. Cerro 1 km. N 52

143. Ojos San Pedro, km. No 21 
144. Curiquincha

145. Ascotán

146. Cebollar 1

147. Cebollar 2

148. Cebollar 3

149. Cebollar 4

150. San Martín

151. Amincha

152. Coposa

153. Yuma

154. Ujina

155. Huasco Lípez

156. Macaya 1

157. Macaya 2

$\begin{aligned} 324 & \text { 193. Mukar } \\ 1.685 & \text { 194. Kori } \\ 228 & \text { 195. Limal (Argentina) } \\ 46 & \text { 196. Guatyquina (Argentina) } \\ 81 & \text { 197. Laguna de Lejía } \\ 462 & \text { 198. Laguna del Laco } \\ 1.754 & \text { 199. Laguna Calca } \\ 434 & \text { 200. Laguna de Tugaktu (orilla Laco) } \\ 19 & \text { 201. Salar Quisquirg } \\ 27 & \text { 202. Tullajeto (Sur Laco) } \\ 174 & \quad \text { Zona Tulán } \\ 25 & \\ 77 & \end{aligned}$

204. Tilomonte: El Molino

Zona Tambillo-Cas-Socaire y Altiplano Chileno

158. Tambillo

159. Alto de Tocolén (Toconao)

206. Miniques

207. Miscanti

160. Zapar (sur ruinas)

744 208. Tilocalar 1

146 209. Tilocalar $1 \mathrm{~b}-\mathrm{c}$

682

325

161. Calarco (Agua Blanca)

210. Tilocalar 2

162. Tumbre

163. Saltar

164. Ana

165. Icura

166. Lascar

167. Cas 1

168. Cas 2

169. Cas 3

170. Cas 4

171. Cas 5

172. Cas 6

173. Cas 7

174. Cas 8

175. Cas 9

176. Cas 10

177. Cas 11

178. Algarrobillo 1

179. Algarrobillo 2

180. Tapus

181. Cuno

182. Hatchar 1

183. Hatchar 2

184. Hatchar sitio

185. Sapaleri Alto

186. Tara: Aguas Calientes

187. Tara: Laguna

188. Tara: Río

189. Laguna límite con Bolivia

190. Pinato (raya argentina $38 \mathrm{~km}$ sur Sapaleri)

191. Leber

192. Leber: Barro Negro

211. Tulán 1

212. Loma 2

213. Loma 3

214. Loma 4

215. Loma 5

216. Loma 6

217. Loma 7

218. Loma 8a I

Tulán

6 232. Loma $13 \quad 35$

219. Loma 8a II 67

220. Loma 8a III $\quad 1.215$

221. Loma 8 a III bis

222. Loma 8a IV $\quad 107$

223. Loma $8 \mathrm{a} \mathrm{v}$

224. Loma 8a VII 155

225. Loma 8a VII $\quad 80$

226. Loma 8 b 94

227. Loma 9

228. Loma 9 bis $\quad 115$

229. Loma $10 \quad 216$

230. Loma $11 \quad 1.225$

231. Loma 12

233. Loma $14 \quad 66$

32 234. Loma 15

69 235. Loma $16 \quad 408$

67 236. Loma 16 bis 24 
237. Loma 17

238. Loma 18

239. Loma 19

240. Loma 20

241. Loma 21

242. Loma 22

243. Loma 23a

244. Loma $23 b$

245. Loma $23 \mathrm{c}$

246. Loma $23 \mathrm{~d}$

247. Loma $23 \mathrm{e}$

248. Loma $23 \mathrm{f}$

249. Loma 24

250. Loma 25

251. Loma 26

252. Loma 27

253. Loma 28

254. Loma 29a

255. Loma 29b

256. Loma 29c

257. Loma 30

258. Loma 30 bis

259. Loma 31

260. Loma 32

261. Loma 33

262. Loma 34

263. Loma 35

264. Loma 36

265. Loma 37

266. Loma 38

267. Loma 39

268. Loma 40

269. Loma 41

270. Loma 42

271. Loma 43

272. Loma 44

273. Loma 45

274. Loma 46

275. Loma 47

276. Loma 48

277. Loma 49

278. Loma 50

279. San Juan Monturaqui

280. Monturaqui (leoncito)
83

136

179

135

225

196

166

104

26

27

348

11

13

Pircas

47

8

Tulán

129

226

137

222

1.947

57 lajas grabadas

88

70

21

74

348

141 lajas grabadas

18

200

55

10

218

45

96

61

183

1.247

4

35

41

44

36

82

259

38
283. Taltal: Camarones

284. Taltal: Punta Morada

285. Taltal: La Caña

286. Paposo

287. Antofagasta

288. Antofagasta: La Chimba

289. Ancachi Sur 331

290. Ancachi Angostura 46

291. Ancachi Centro 293

292. Quillagua: Cordillera 365

293. Mamiña (Tarapacá) 28

294. Caserones (Tarapacá: Núñez) 20

295. Soronal (Tarapacá: Núñez) 25

296. Punta Pichalo (Pisagua) 184

297. Parapallo Yaluga (Pisagua Altiplano) 15

Arica

298. Camino de Paquisa a Caracoto

299. Guayatiri (Altiplano frontera con

Bolivia)

300. Sora Sora

10

1

Bolivia

Zona Soniquera (Zuniquera)

301. Soniquera (pueblo)

145

302. Soniquera bajo 47

303. Campo Blanco 71

304. Puerta y Hornillos 357

305. Quebrada Ramaditos 38

306. Río Salado 29

307. Quebrada Horno 48

308. Pavillón 84

309. Río Soniquera 106

310. Cancha Maya 48

311. Juntas 37

312. Chalviri 124

313. Chanillas 1

314. Chanillas 2

315. Potrero 300

316. Río Volcán 445

317. Cruz Vinto 240

318. Hornillos 689

319. Huellas 85

320. Agua Chica 26

321. Todos Santos 59

322. Loroni (30 km al este de Soniquera) 207

323. Ojito ( $1 \mathrm{hr}$. al poniente de Todos Santos) 33

\section{Zona Ketena}

57 324. Ketena (pueblo)

?

21 325. Cuevas
8

1

44

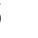

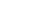

(n)

28

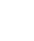

5

15

15

3

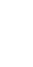

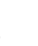

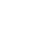

87

24

$(228$

0

0

689

85

26

9


326. Batea

327. Pampa de Batea

328. Quebrada Seca

329. Lagunita

330. Laguna Morijón

331. Río Ketena

332. Buena Vista (Wina Vista?)

333. Silvaquera

334. Ojos de Katabies (Katari)

335. Cerro Sanadori

336. Ciénaga Grande

337. Tchuncal

338. Calcanis

339. Puntita Negra (Puntas Negras)

340. Fundición

341. Potrero

342. Tío Craces

343. Redonda

344. Torquis

345. Tchasquilla 1

346. Tchasquilla 2

347. Tchasquilla 3

348. Tchasquilla Ara Loma

349. Tchasquilla Zapa Viejo

350. Cueva Lázaro

351. Morrito Blanco

352. Temblorcito

353. Ghatchi

354. Punta Grande

355. Mayte Gara (Huytecara)

356. Yuntacha

357. Peña Barrosa

358. Sol de la mañana

359. Tolapamba ( $8 \mathrm{~km}$ al este de Ciénaga)

360. Walcaro (oriente de la apacheta camino a Ketena)

361. Entre Ketena y San Antonio Tchengua

362. Sur de Ketena (pampa Cara)

263. 25 km sur de Ketena, Isco

364. Sur de Ketena, Isco Cueva arriba

365. Este de Ketena: Aguas Calientes

$366.40 \mathrm{~km}$ este de Ketena: Castor

367. Ketena Chico (pueblo)

368. Ketena Chico: Río Lípez

369. Ketena Chico: Místico

\section{San Antonio de Lípez}

370. San Antonio de Lípez

371. Canchilla

372. Swisquis
162

44

1

122

256

59

48

195

149

31

16

42

19

26

898

17

121

10

89

26

6

8

43

10

17

160

5
373. Aguas Calientes 136

374. Rodríguez 48

375. Cuevas Campos 14

376. Cuevas 184

377. Pavillón (Pabellón) 35

San Agustín

378. San Agustín 205

379. Caracoles $\quad 1.102$

380. Cevenka 8

381. Arlota 171

382. Todos Santos 93

383. Herquilla (Cerro Cevenka) 17

384. Laguna Colorada 5

385. Laguna Colorada Sur 28

386. Laguna Colorada Este $(1 \mathrm{~km}) \quad 18$

387. Laguna Colorada Sulor 48

388. Ciénaga 48

389. Torquis 10

390. Conajatanís 11

391. Guaya Cara 11

392. Laguna Hedionda $\quad 140$

393. Laguna Verde 42

394. Laguna Verde: Aguas Blancas 22

395. Laguna Verde: Aguas Calientes 17

396. Wiraska (2 días al norte de Laguna Verde)

397. San Juan 116

398. San Juan: Amor 13

399. San Pablo de Suslípez 43

400. San Pedro de Quemez (Norte Ollahue) 82

401. Catal 30

402. Catal: Laguna de Yape 14

403. Matil 2

404. Guallagua 18

Totales por zonas

1. Salitre

59.473

2. Talabre-Loa 46.823

3. Tchasquir 231

4. Ghatchi 28.259

5. Chaxas 7.897

6. Loma Negra (Puripica-Guatin) $\quad 7.897$

7. Zona Ghatchi-Loma Negra-Guatin 5.693

8. Zona Norte (Centro, Salares Norte) 25.650

9. Tambillo y zona $\quad 16.429$

10. Zona Tulán 2.636

11. Tulán 23.649

12. Monturaqui 297

13. Zona costera $\quad 1.938$ 
Total material lítico conservado en Museo 296.154

Material lítico conservado en el

Museo de proveniencia desde Bolivia

Donaciones de muestras de material lítico al Museo de San Pedro de Atacama

Chile:

Magallanes

Isla de Pascua

2

Perú:

Oquendo

Chivateros

Argentina:

El Toro

Tres Morros

Poronguillos (Los Zazos)

Saladenos (El Cajón)

Pabellón Chico (Valle Cajón)

Santa Cruz

Barranea

Turi Lari I

Turi Lari II

Yavi I

Yavi II

Patagonia

Venezuela

\section{A. Lista de hábitats, pueblos, estancias}

1. Paleolítico.

Ghatchi 2 (sitio norte)

Ghatchi 1

Puripica

Guatin (oriente)

Algarrobillo 1

Miniques

Tumbre

Calarcoco

San Martín

Algarrobillo 2

Ojos de San Pedro

Ghatchi loma 28 bis

Chaxas (sitio oriente)

Ghatchi 2 (sitio meridional)

Tulán 1

Tapus 1

Lari
Miscanti

Zapar (sur)

San Juan de Monturaqui

Cueva de la Damiana

2. Mesolítico.

Machuca

Hatchar (Socaire)

Tilocalar 3

Guatin

Cuno (Socaire)

Hatchar 2 (Socaire)

Socaire (pueblo)

3. Neolítico.

Calar

Tapus 2

Nepi (Socaire)

Tilocalar 1

Algarrobillo (Valle)

Alto de Labra 2

Calarcoco (Santa Ana)

Cuesta de San Bartolo

Lasana

Pucara de Quitor

Oyirinitor (Toconao)

Pamiri

Peine (ruinas pueblo)

Quihusuma (Socaire)

Vilama 2

Solor 4

Coyo

Tkiu-Tchu

Talikuna (Caspana)

Tchapuraqui

Tilocalar 2

Alto de Labra 1

Alto de Tocolén

Catarpe

Hatchar 3

(Turi)

Lava

Paguaytate (Topain)

Peine (oriente)

Soncor

Vilama 1

Zapar

Tulor

Toconao

Socaire

Camar 
4. Epoca Incásica.

Catarpe: Tambo

(Chiu Chiu: Pucara)

Oyrintor

Sitios votivos.

Lincabur

Pular

Cerro Colorado

Miño

Llullayaco

Catarpe Refugio

Licancaur: centro religioso y pueblo

Pili

Yariques

San Pedro y San Pablo

Socompa

5. Estancias.
Ales (Talabre)
Calarcoco
Peñaliri (Río Grande)
Salitre (Río Grande)
Talabre
Ana (Talabre)
Junta (Río Grande)
San Juan (Río Grande)
Ocara (Toconao)
Tebenquiche

6. Colonial.

Beter (San Pedro de Atacama)

\section{B. Lista de lugares con petroglifos-pinturas rupestres}

1. Pinturas rupestres.

Cueva de la Damiana

Cueva del Río Salado

Cuevita Quebrada occidental de Toconao

2. Petroglifos.

Cueva de la Damiana (interior y exterior)

Cueva de Tulán

Cueva de Tupe

Tulán (Peña norte del río)

Confluencia ríos Caspana con el río Salado

Curte (Caspana)

Tchapuraqui (Toconao)
Taira (Loa)

Santa Bárbara (Loa)

Quebrada de Queri (Toconao)

Calama (río Loa) destruidos

Catarpe 3 sitios

Tamor

Lasana Chiu Chiu

Poma

Toconce

Río Salado

Soncor

Quebrada de Calar

Tropero de San Pedro a Río Grande:

1 San Bartolo, 2. Ayaviri y 3. Cerro Blanco

Quebrada de Caire

Quebrada de Tambores: 4 sitios

Caspana

Tononkos (Toconao)

N.B. Lista de sitios sin tomar en cuenta los estilos, la naturaleza de los dibujos, el simbolismo o el naturalismo, etc.

3. Lista de los cementerios descubiertos y estudiados con el índice cefálico de los cráneos para los cuales se tomaron en cuenta únicamente aquellos de adultos que no han sido destruidos o deformados artificialmente.

El cuadro blanco significa el término medio.

Las líneas negras indican que los cementerios están fuera de la zona de San Pedro de Atacama.

Cada cementerio tiene hecho un estudio, cráneo por cráneo, con su diagrama completo.

En el primer cuadro damos solamente un resumen de todo el conjunto de cráneos. En el segundo cuadro damos un estudio de un cementerio (Catarpe 2) con su proporción de cráneos deformados, mortalidad infantil, etc.

Todos los demás cementerios tienen un estudio igual a éste.

\section{Lista del material arqueológico del Período} Neolítico expuesto en las salas del museo.

Cántaros (todo tipo)

Urnas enterradas

Urnas quebradas

Fragmentos de cerámica grabados

millares

Fragmentos de cerámica pintados 
Tabletas para rapé

Tubitos

Espátulas

Morteritos

Vasitos

Canastas (todo tipo)

Gorros

Pipas

Objetos de Cobre
$443 \quad$ Collares

143

394 Adornos (todo tipo)

384 Calabazas pirograbadas

856

182

198

290

253

99

119

402
Tembetás

302

Cucharas de madera 192

Hachas de piedra 221

Rompe cabezas (mazas)

49

1.500

cc. 60.000 


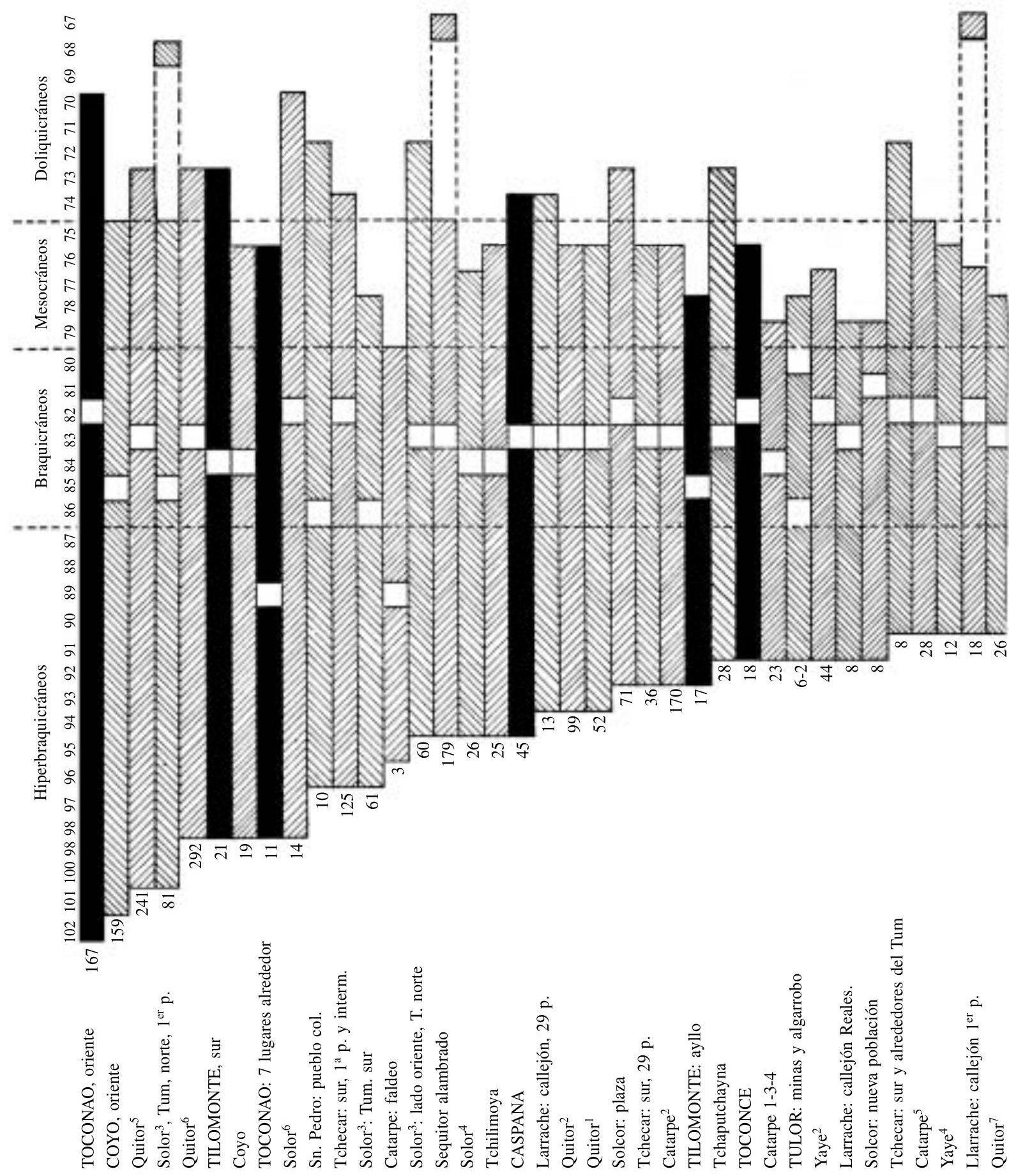



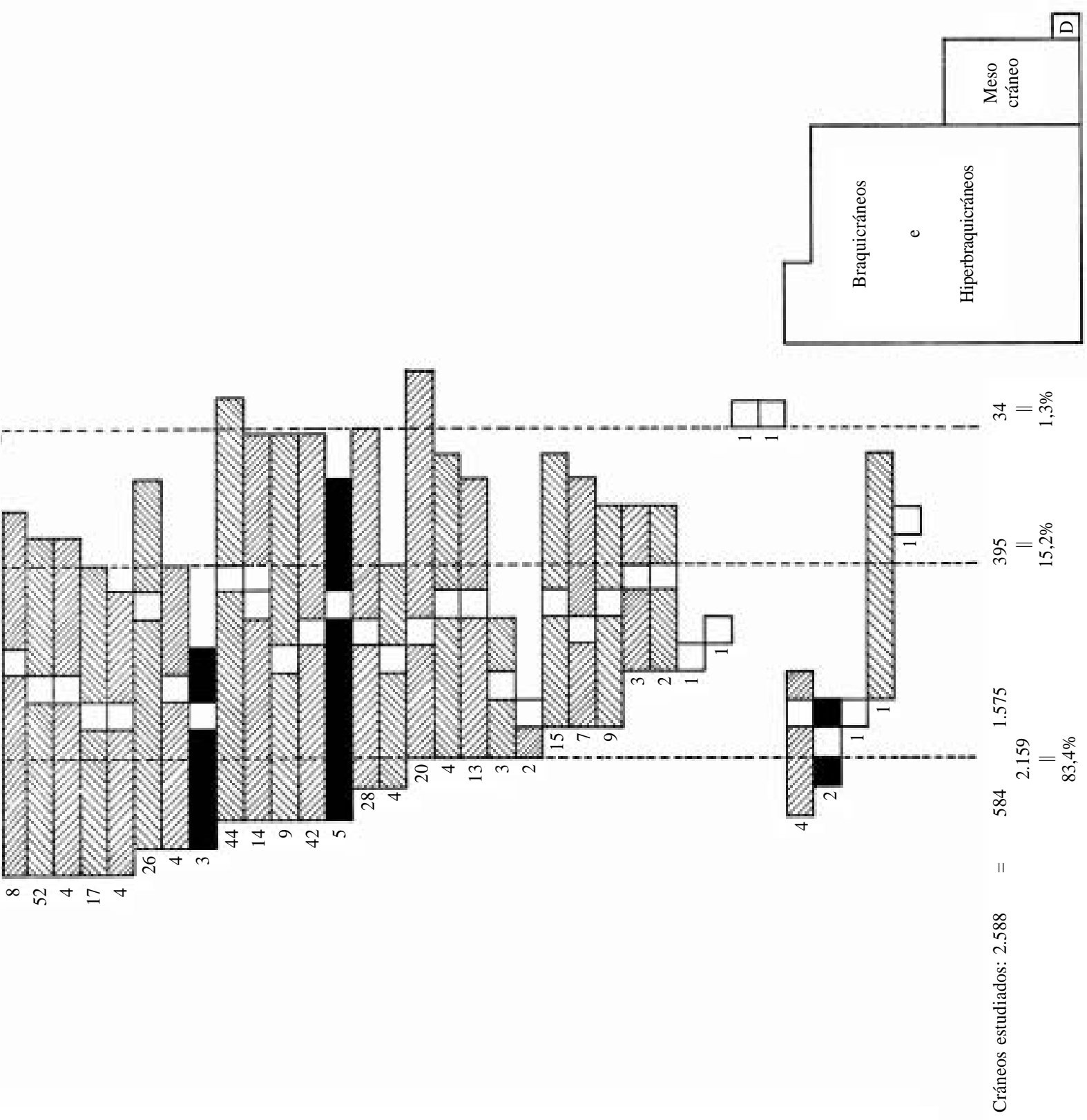

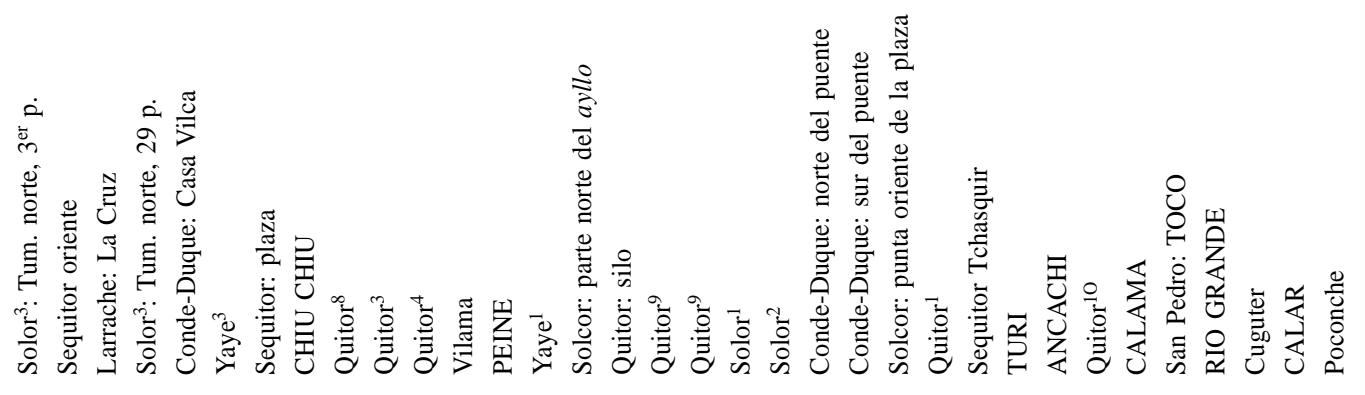




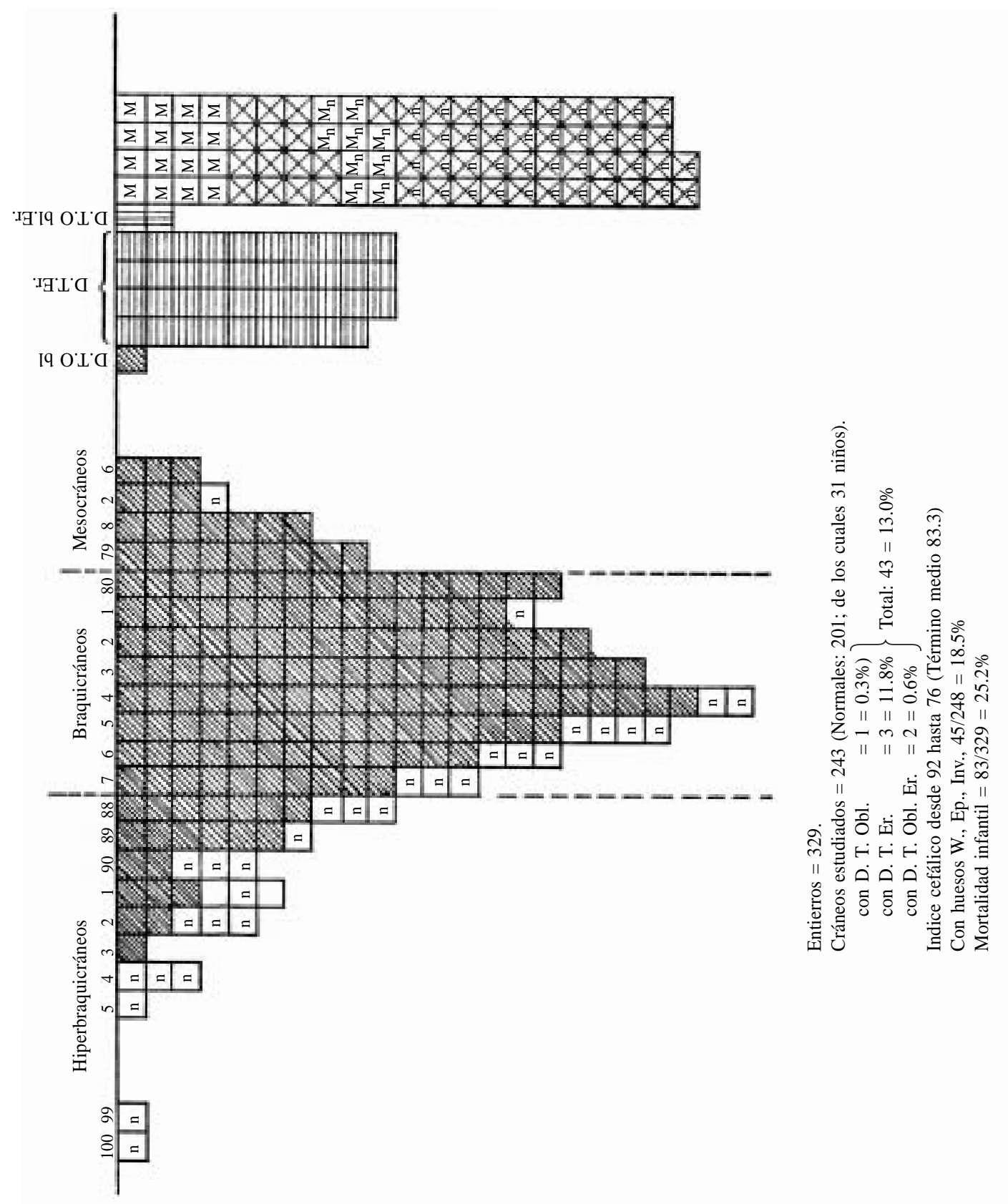

